\title{
CHARACTERISTICS OF PLASMAS PRODUCED BY DOUBLE BEAT WAVE INTERACTION IN THE NEPTUNE FACILITY AT UCLA*
}

\author{
R. Narang ${ }^{\dagger}$, C. Filip, S. Ya. Tochitsky, D. Gordon ${ }^{\ddagger}$ C. E. Clayton, K. A. Marsh, C. Joshi \\ UCLA Electrical Engineering Department, Los Angeles, CA 90095
}

\begin{abstract}
Cathodeless electron sources have received a great deal of attention recently. In a similar scheme for generating a high current relativistic electron beam described here, two beat waves are used: one to setup an accelerating structure, and another to locally perturb this structure in order to trap background electrons from the resonant density plasma. This is called the "Double Beat Wave Injector" scheme. We report the characterization of plasmas formed by the superposition of two beating electromagnetic wave pairs with peak intensities greater than the tunneling ionization threshold for hydrogen. The plasmas produced by the beat waves are diagnosed using optical imaging and timeresolved Thomson scattering, separately as well as when both beat waves interfere with each other. The brightness of the plasma is maximum near the resonant fill pressure, as expected. Preliminary results are presented.
\end{abstract}

\section{MOTIVATION}

A variety of all-optical methods have been proposed for generating a high current beam of relativistic electrons for injection into an accelerating structure [1,2]. Here we propose an alternate scheme for a cathodeless or an all-optical injector which is called the "Double Beat Wave Injector" (DBWI) scheme. Relativistic electron plasma waves can be driven by beating electromagnetic waves where the difference between the laser frequencies, $\Delta \omega=\omega_{2}-\omega_{1}$, is equal to the plasma frequency $\omega_{\mathrm{p}}$. In the past these beat excited relativistic plasma waves have been used to accelerate externally injected electrons [3]. In the DBWI scheme one beating electromagnetic wave from a two-wavelength laser pulse drives a relativistic electron plasma wave (driving pulse), while a second copropagating beating electromagnetic wave perturbs this wave (perturbing pulse). Local constructive interference of the two plasma waves results in self-trapping of electrons. To allow the acceleration of self-trapped electrons over a long distance it is of importance that the driving pulse produces a plasma wave significantly longer than that produced by the perturbing pulse.

\subsection{Simulation of the DBWI Scheme}

The potential viability of the DBWI scheme was explored in 2-D modeling done using TURBOWAVE [4] which is a code that treats the electrons as particles with the lasers acting on the electrons only through the ponderomotive force. TURBOWAVE has the ability to model the motion of individual electrons, but with the limitation that electron orbits do not cross as is the case when trapping occurs. The code includes tunneling ionization and refraction. The DBWI scheme relies on wave-breaking through localized trapping as the source of injection electrons. Therefore the code is able to provide adequate information about the field structure up to the point where trapping occurs. The simulation was done over a distance of $20 \mathrm{~mm}$ with both beat waves in phase for hydrogen gas parameters. The laser parameters used in the simulation are close to the experimental values. The normalized laser electric field amplitude, generally defined as $\alpha=\mathrm{eE} / \mathrm{mc} \omega$ (where $\mathrm{E}$ is the laser electric field and $\omega$ is the laser frequency), is taken to be 0.5 for the perturbing pulse and 0.2 for the driving pulse. The risetime of both laser pulses is $100 \mathrm{ps}$, with $\Delta \omega=\omega_{\mathrm{p}}$.

Figure 1 shows a contour plot of the longitudinal electric field $E_{x}$ of the plasma wave as a function of distance after $120 \mathrm{ps}$.

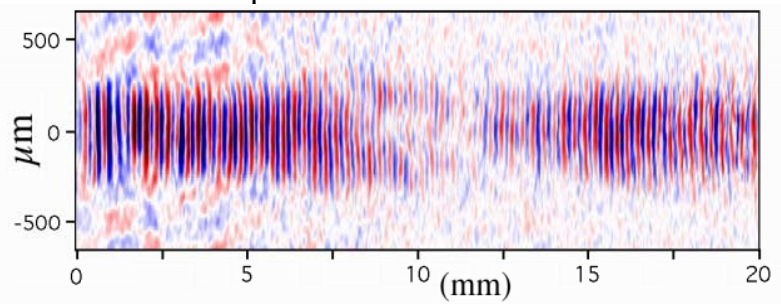

Figure 1: Contour plot of the longitudinal electric field $E_{x}$ as a function of axial distance in $\mathrm{mm}$.

Local wave-breaking can be seen at the center of the simulation window which corresponds to the focus of the lasers. In this region wave-breaking is manifested as damping of the plasma wave amplitude. The longitudinal electric field at the point before the onset of wave-breaking reaches a maximum of $0.8 \mathrm{E}_{0}$, where $\mathrm{E}_{0}=\mathrm{mc} \omega_{\mathrm{p}} / \mathrm{e}$ is the normalized cold-plasma wave-breaking amplitude. This indicates a possibility for experimental observation of electrons in the forward direction. Electron energies near $\mathrm{MeV}$ levels are expected.

\section{EXPERIMENTAL SETUP}

The experiment is being done at the Neptune Laboratory at UCLA [5] using a terawatt $\mathrm{CO}_{2}$ laser system. This system is able to produce two-wavelength

\footnotetext{
*Supported by DOE grant DE-FG03-92ER40727

† ritesh@ucla.edu

$\doteqdot$ currently at Naval Research Labs
} 
pulses at $10.6 \mu \mathrm{m}$ and $10.3 \mu \mathrm{m}$ with nearly equal line ratios. In Fig. 2 the layout of the experiment is presented. The experiment utilizes two beams originating from the same output beam from the Neptune laser with a pulse length of $\sim 120 \mathrm{ps}[6]$. A 2" mirror is used to split the central part of the laser beam and send it to an $f / 3$ offaxis parabolic mirror (OAP) which provides a spot size $\mathrm{w}_{0} \approx 80 \mu \mathrm{m}$, and an intensity of $8 \times 10^{14} \mathrm{~W} / \mathrm{cm}^{2}$ (perturbing arm). The outer portion of the beam is sent to an $\mathrm{f} / 18$ $\mathrm{NaCl}$ lens giving a spot size $\mathrm{w}_{0} \approx 200 \mu \mathrm{m}$ and an intensity of $4 \times 10^{14} \mathrm{~W} / \mathrm{cm}^{2}$ (driving arm). The $\mathrm{f} / 18$ beam passes through a hole in the OAP so the two beams propagate collinearly.

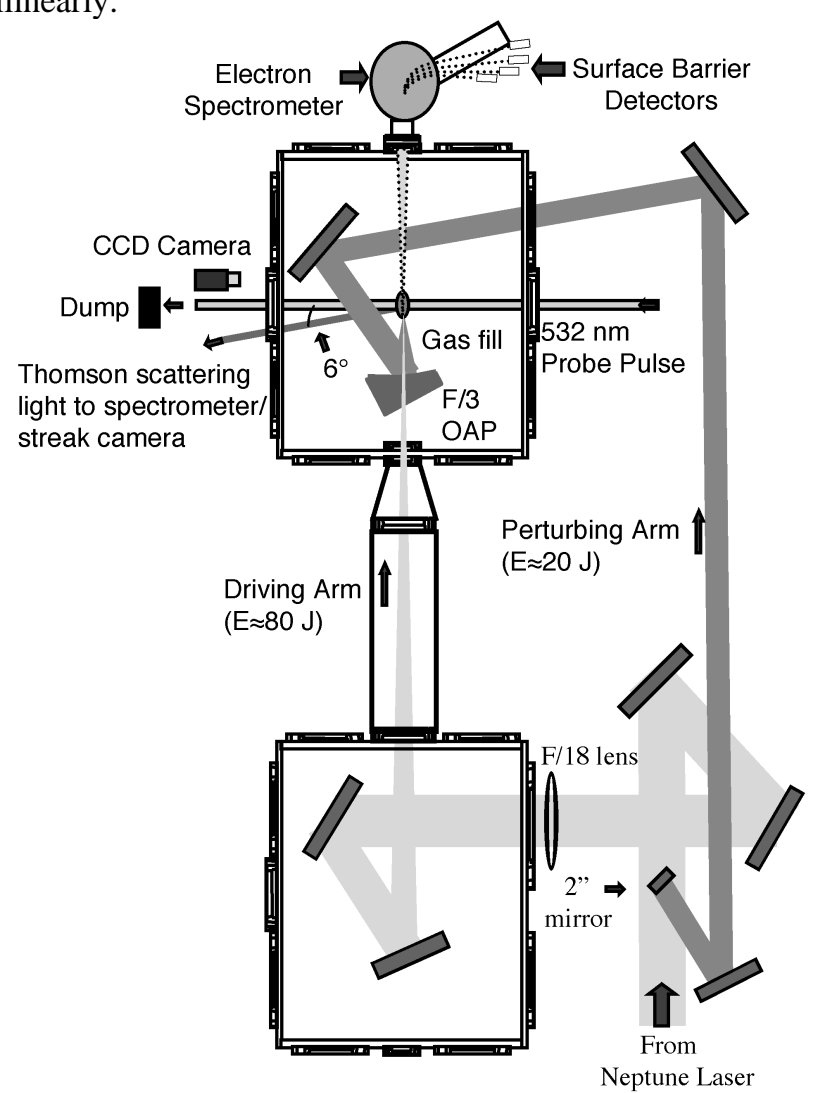

Figure 2: Setup for the DBWI experiment.

The two beams are spatially overlapped using an IR imaging system. Fine adjustment of the alignment of the two arms was done using magnified camera images with $40 \mu \mathrm{m}$ resolution. Plasmas are produced in hydrogen gas for both focusing geometries at pressures corresponding to resonant plasma beat wave excitation ( 160 mtorr). The plasmas are viewed using 8-bit CCD cameras and a typical image is presented in Fig. 3. The plasmas produced by the $f / 18$ and $f / 3$ beams have Rayleigh ranges of $2 \mathrm{z}_{\mathrm{R}} \approx 25 \mathrm{~mm}$ and $2 \mathrm{z}_{\mathrm{R}} \approx 4 \mathrm{~mm}$, respectively. Clearly both plasmas are spatially overlapped. The light that is orthogonal to the beam propagation in Fig. 3 is attributable to secondary electrons producing fluorescence in hydrogen.
The electron diagnostic used is an electron spectrometer consisting of a dipole magnet with Browne and Buechner pole pieces and surface barrier detectors. This spectrometer is able to image electrons produced at the laser focus and detect electrons in the range of $0.2-30 \mathrm{MeV}$. Electrons are detected using $1 \mathrm{~mm}$ thick, biased silicon surface barrier detectors. With the current setup electrons with a minimum energy of $200 \mathrm{keV}$ are detectable.

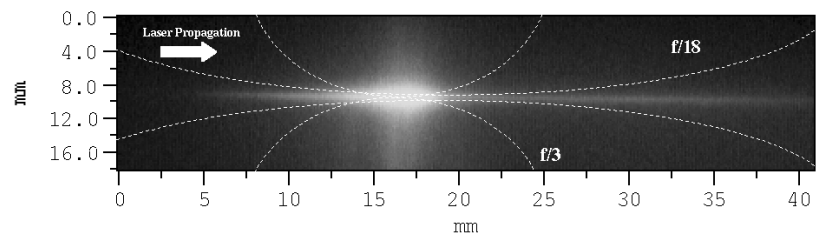

Figure 3: Plasmas in 165 mtorr of $\mathrm{H}_{2}$ produced by the $\mathrm{f} / 18$ and $\mathrm{f} / 3$ arms (dotted lines indicate beam envelopes).

\section{PULSE SYNCHRONIZATION AND DETERMINATION OF RESONANT DENSITY}

Time-resolved Thomson scattering [7] is used for the synchronization of both optical arms. The scattering geometry used (Fig. 2) allows the detection of waves attributable to ion and electron plasma waves. Plasma waves for both $f / 3$ and $f / 18$ arms were probed by a $532 \mathrm{~nm}$ beam and Thomson scattering spectra were observed on a streak camera.
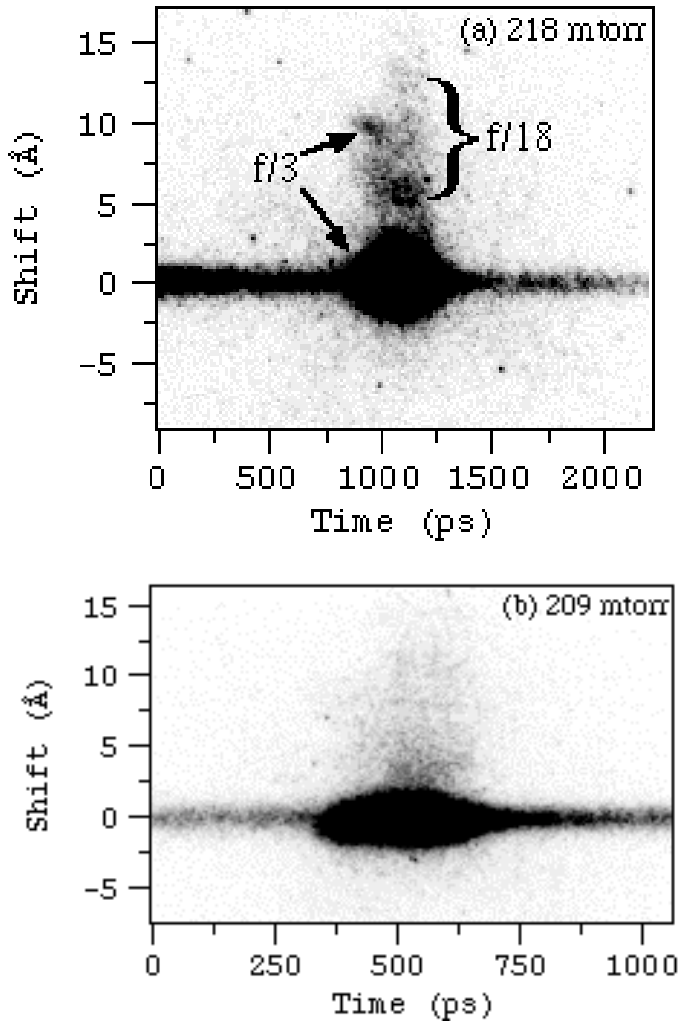

Figure 4: Thomson scattering spectra produced by $\mathrm{f} / 3$ and $\mathrm{f} / 18$ arms in $\mathrm{H}_{2}$ (a) without, and (b) with synchronization. 
Figure 4(a) presents the time-resolved images of the Thomson scattered light when the $\mathrm{f} / 18$ driving pulse follows the $\mathrm{f} / 3$ perturbing pulse by $\sim 90 \mathrm{ps}$. The characteristic features of each arm are different which greatly simplified the synchronization procedure. The plasma waves created by the $f / 3$ beam produce a feature characteristic of Brillouin scattering unshifted in wavelength, as well as a feature characteristic of Raman scattering shifted by $\omega_{\mathrm{p}}$. The plasma created by the $\mathrm{f} / 18$ arm, conversely, produces a broadband signature which is more characteristic of Compton scattering [8]. As shown in Fig. 4(b), when both beat waves are synchronized the Brillouin scattering feature strongly dominates the observed spectrum. However, a slight change in timing of the two pulses on the order of $10 \mathrm{ps}$ immediately results in the appearance of all characteristic features. It should be noted that timing was adjusted simply by translating the 2" beam splitting mirror. The Thomson scattering method allows pulse synchronization on a $10 \mathrm{ps}$ timescale.

The pressure corresponding to the resonant density is determined by measuring the shift in the Thomson scattered spectra of the Raman component of the scattered light (see Fig. 4(a)). Figure 5 presents the linear scaling between the wavelength shift and the square root of the hydrogen gas pressure. Zero shift corresponds the $532 \mathrm{~nm}$ probe wavelength and an $8 \AA$ shift corresponds to $\Delta \omega=\omega_{\mathrm{p}}$ which occurs at a pressure of $\sim 165$ mtorr. DBWI experiments have been done around this pressure.

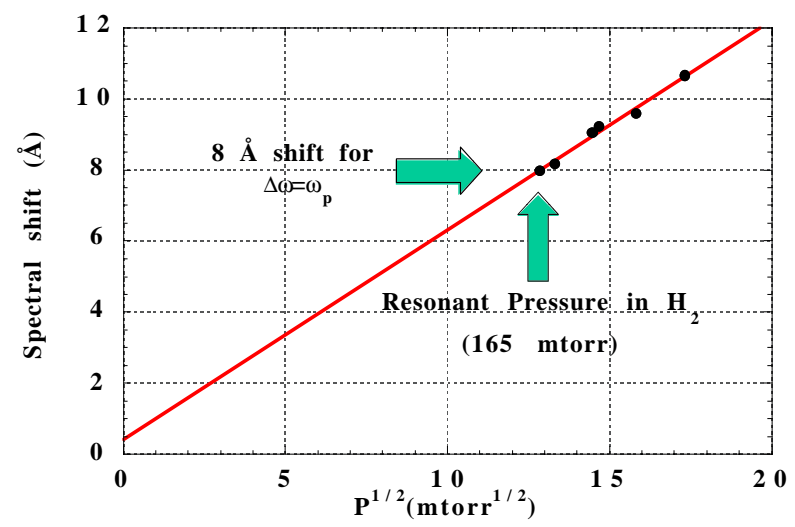

Figure 5: Spectral shift of the Raman component in $\mathrm{H}_{2}$ of the Thomson scattered light versus square root of pressure.

At resonant pressure plasma brightness increases significantly under beat wave illumination conditions and is illustrated in Fig. 6(a). Moreover, plasma brightness strongly depends on the line ratio between the $10.6 \mu \mathrm{m}$ and $10.3 \mu \mathrm{m}$ wavelengths, as seen from Fig. 6(b). The observed brightness of the plasmas is greatest when line ratios are near unity. Therefore, plasma brightness may be indicative of the existence of the relativistic plasma wave.
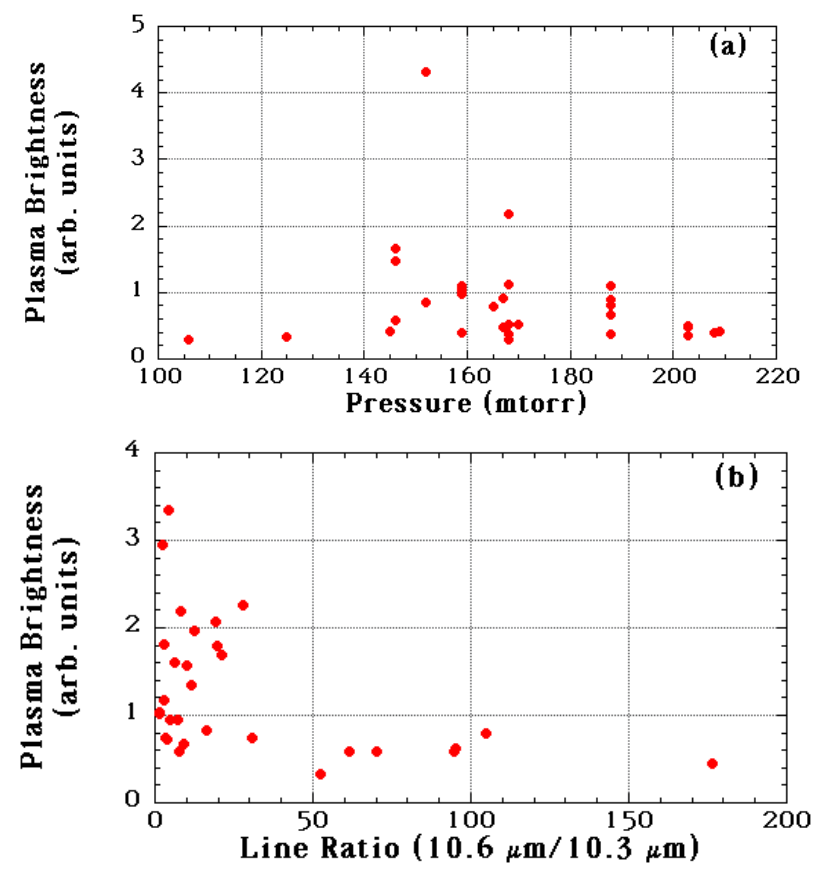

Figure 6: Plasma brightness in $\mathrm{H}_{2}$ (a) versus pressure and (b) versus line ratio.

\section{STATUS AND CONCLUSION}

Laser-plasmas of the appropriate density have been produced and have been synchronized in space to $40 \mu \mathrm{m}$ and in time to $10 \mathrm{ps}$ for both the perturbing arm (f/3) as well as the driving arm (f/18) for the DBWI scheme. To date no electrons have been observed using the Double Beat Wave Injector scheme. Current efforts are directed toward measuring the spatial structure of the relativistic plasma wave to determine whether localized wave-breaking can occur.

\section{REFERENCES}

[1] D. Umstadter et al., Phys. Rev. Lett. 76, 2073 (1996).

[2] C.I. Moore et al., Phys. Rev. Lett. 82, 1688 (1999).

[3] M. Everett et al., Nature 368, No. 6471, 527 (1994).

[4] D. Gordon, Ph.D. dissertation, University of California, Los Angeles, 1999.

[5] C. E. Clayton et al., Nuc. Inst. Meth. 410, No. 3, 378 (1998).

[6] S. Ya. Tochitsky et al., Opt. Lett. 26, No. 11, 813 (2001).

[7] A. Lal et al., Phys. Plasmas 4, No.5, 1434 (1997).

[8] W. P. Leemans et al., Phys. Rev. Lett. 67, 1434 (1991). 